OPEN ACCESS

Edited by:

Marsida Kallupi,

University of California, San Diego,

United States

Reviewed by:

Shelley Warlow,

UC San Diego Health, United States

Reesha Patel,

Salk Institute for Biological Studies,

United States

Yarimar Carrasquillo,

National Institutes of Health (NIH),

United States

*Correspondence:

J. Andrew Hardaway

andrewhardaway@uabmc.edu

Specialty section:

This article was submitted to

Motivation and Reward,

a section of the journal

Frontiers in Behavioral Neuroscience

Received: 11 June 2021 Accepted: 06 December 2021 Published: 24 December 2021

Citation:

Zeng N, Cutts EJ, Lopez CB, Kaur S,

Duran M, Virkus SA and Hardaway JA (2021) Anatomical and Functional

Characterization of Central Amygdala Glucagon-Like Peptide 1 Receptor Expressing Neurons.

Front. Behav. Neurosci. 15:724030. doi: 10.3389/fnbeh.2021.724030

\section{Anatomical and Functional Characterization of Central Amygdala Glucagon-Like Peptide 1 Receptor Expressing Neurons}

\author{
Ningxiang Zeng, Elam J. Cutts, Christian B. Lopez, Simran Kaur, Miguel Duran, \\ Sonja A. Virkus and J. Andrew Hardaway*
}

Department of Psychiatry and Behavioral Neurobiology, University of Alabama at Birmingham, Birmingham, AL, United States

Glucagon-like peptide 1 receptors (GLP-1Rs) are highly expressed in the brain and are responsible for mediating the acute anorexigenic actions of widely prescribed GLP-1R agonists. Neurobiological efforts to localize the hypophagic effects of GLP-1R agonists in the brain have mainly focused on the hypothalamus and hindbrain. In this study, we performed a deep anatomical and neurophysiological characterization of GLP-1Rs in the central nucleus of the amygdala (CeA). At an mRNA level, we found that Glp1r is diffusely coexpressed in known CeA subpopulations like protein kinase c $\delta$ (Prkcd), somatostatin (Sst), or tachykinin2 (Tac2). At a cellular level, we used G/p1r-Cre mice and viral Cre-dependent tracing to map the anatomical positions of GLP-1R cells across the rostral-caudal axis of the CeA and in CeA subdivisions. We found that G/p $1 r^{\mathrm{CeA}}$ cells are highly enriched in the medial subdivision of the CeA (CeM). Using whole cell patch clamp electrophysiology, we found that G/p $1 r^{\mathrm{CeA}}$ neurons are characterized by the presence of $I_{h}$-like currents and resemble a low threshold bursting neuronal subtype in response to hyperpolarizing and depolarizing current injections. We observed sex differences in the magnitude of $I_{h}$-like currents and membrane capacitance. At rest, we observed that nearly half of G/p $1 r^{\mathrm{CeA}}$ neurons are spontaneously active. We observed that active and inactive neurons display significant differences in excitability even when normalized to an identical holding potential. Our data are the first to deeply characterize the pattern of G/p1r in the CeA and study the neurophysiological characteristics of CeA neurons expressing G/p1r. Future studies leveraging these data will be important to understanding the impact of GLP-1R agonists on feeding and motivation.

Keywords: central amygdala (CeA), anatomy, neural circuit, electrophysiology, glucagon-like peptide 1 receptor (GLP-1R), glucagon-like peptide 1 (GLP-1)

\section{INTRODUCTION}

Obesity is a growing health problem for developed nations. In the United States alone, the CDC estimated that obesity prevalence among adults increased from 30.5 to $42.4 \%$ over the last 18 years indicating more than a third of adults in the United States are obese (BMI > 30). With obesity comes an increased risk for chronic diseases including cardiovascular disease, cancer, and type II diabetes. Many individuals with type II diabetes are prescribed drugs that target the glucagon-like 
peptide 1 receptor (GLP-1R) due to the clinical benefit of enhancing the release of insulin while also decreasing appetite (Müller et al., 2019). Despite their wide use, a complete understanding of the full biological and behavioral impacts of GLP-1R agonism is lacking.

When administered via intracerebroventricular (icv) injection, GLP-1R agonists elicit hypophagia indicating that neuronal GLP-1Rs are required for this hypophagic effect. Thus, the anorexigenic effect of GLP-1R agonists can be attributed to the activation of neuronal not peripheral GLP-1Rs (Burmeister et al., 2013, 2017; Secher et al., 2014; Sisley et al., 2014; Adams et al., 2018). Attempts to localize and attribute the actions of GLP-1R signaling on feeding to specific brain nuclei have focused mainly on hypothalamic and hindbrain GLP-1Rs known to control hunger states or meal termination (Hayes et al., 2009; Kanoski et al., 2011; Alhadeff et al., 2014). However, the function of GLP-1Rs in limbic sites-like the lateral septum (LS), bed nucleus of the stria terminalis (BNST), and the central amygdala (CeA) - likely plays a key motivational role in feeding. Of relevance, recent work has shown that BNST GLP-1Rs and GLP-1R-expressing cells may in part drive stress-induced hypophagia and that LS GLP-1Rs functionally regulate feeding and motivation (Terrill et al., 2016; Williams et al., 2018). In the CeA, however, very little is known about how GLP-1Rs regulate either feeding or motivation.

The CeA is a complex heterogenous structure that contains both orexigenic and anorexigenic subpopulations of neurons. These neuronal subpopulations regulate emotional and survival behaviors including responses to predators and noxious stimuli (Haubensak et al., 2010; Li et al., 2013; Janak and Tye, 2015; Douglass et al., 2017; Hardaway et al., 2019; Ip et al., 2019; Steinberg et al., 2020; Weera et al., 2021). Previous studies using mice and rats have identified the CeA as a site for GLP-1R expression; however, the pattern of GLP-1R expression within a framework of known CeA populations has been unclear. In this study, we performed a deep anatomical characterization of CeA GLP-1Rs and GLP-1R-expressing neurons and utilized slice electrophysiology to characterize the membrane and neurophysiological properties of Glp $1 r^{\mathrm{CeA}}$ neurons.

\section{MATERIALS AND METHODS}

\section{Animals}

We used 8-16 week old C57BL6/J (Jackson Labs, Bar Harbor, ME) or Glp1r-Cre (MGI ID: 5776617) male and female mice for this study (Williams et al., 2016). For all experiments, animals were group housed 3-5/cage. Mice were maintained on a standard 12:12 light cycle with lights on at 7 a.m. Unless otherwise indicated, food and water were provided ad libitum. All procedures were performed according to an approved animal protocol by the University of Alabama at Birmingham Institutional Animal Care and Use Committee.

\section{Stereotaxic Surgery}

Survival surgeries were performed on 6-8 week old Glp1r-Cre mice. On the day of the surgery, animals received $0.1 \mathrm{mg} / \mathrm{kg}$ Buprenorphine SR (sc) and $5 \mathrm{mg} / \mathrm{kg}$ Meloxicam SR (sc). Under isoflurane inhalation $(0.5-5 \%)$, mice were placed in a stereotaxic frame. The scalp was depilated and sterilized using rotating $70 \% \mathrm{EtOH}$ and betadine application. Topical lidocaine (4\%) and triple antibiotic were then applied to the scalp in preparation for incision. After ensuring a deep plane of anesthesia via loss of toe pinch reflex, a midline incision was made to expose the skull surface. A craniotomy was then performed at the injection site above the CeA. AAV5-hSyn-DIO-mCherry (300 nl, Addgene - lot\# v63478) was injected at $-1.30 \mathrm{~mm}$ posterior, $\pm 2.90 \mathrm{~mm}$ lateral, and $-4.60 \mathrm{~mm}$ ventral of bregma. Mice remained in the colony for 3-4 weeks following surgery for post-operative recovery and to allow accumulation of mCherry expression in the CeA prior to perfusion or electrophysiological recordings.

\section{Histology}

Glp1r-Cre mice were injected with a terminal dose of tribromoethanol $(250 \mathrm{mg} / \mathrm{kg})$. After reaching a deep plane of anesthesia confirmed via loss of toe pinch reflex, animals were transcardially perfused with $0.01 \mathrm{M}$ PBS and then $50 \mathrm{ml}$ of $4 \%$ paraformaldehyde in $0.01 \mathrm{M}$ PBS. Brains were extracted and post-fixed in $4 \%$ paraformaldehyde/PBS overnight then transferred to $30 \%$ sucrose for cryoprotection for $36-48 \mathrm{~h}$ prior to cryosectioning. Free floating sections were mounted to Superfrost plus microscope slides (Fisher Scientific; Waltham, MA) and coverslipped with mounting media containing DAPI (Vector Laboratories).

\section{Fluorescence in situ Hybridization}

Fresh tissue was harvested from 8 to 16 week old C57BL6J or Glp1r-Cre male and female mice and flash frozen on dry ice then stored at $-80^{\circ} \mathrm{C}$. Brains were cryosectioned at $20 \mu \mathrm{M}$ and directly mounted onto Superfrost Plus slides (Fisher Scientific; Waltham, MA) then stored in a sealed container at $-80^{\circ} \mathrm{C}$. The single molecule RNAscope fluorescent multiplex assay was performed according to the manufacturer's protocol (Advanced Cell Diagnostics; Newark, CA). The target probe for Glp1r was applied in tandem with the appropriate probe for Prkcd, Sst, Tac2, or Cre. For each RNAscope multiplex florescent assay, the target probe for Glp1r was assigned to $647 \mathrm{~nm}$ excitation while target probes for Prkcd, Sst, Tac2, and Cre were assigned to $550 \mathrm{~nm}$ excitation using the appropriate amplification buffers. Appropriate negative controls from the manufacturer were included for each assay for verification of probe specificity. The following target probes were used for this manuscript:

$\begin{array}{ll}\text { 446391-Mm-Tac2 } & \text { Accession \#: NM_009312.2 } \\ \text { 404631-Mm-Sst } & \text { Accession \#: NM_009215.1 } \\ \text { 441791-Mm-Prkcd } & \text { Accession \#: NM_011103.3 } \\ \text { 418851-C3-Mm-Glp1r } & \text { Accession \#: NM_021332.2 } \\ \text { 312281-Cre } & \text { Accession \#: KC845567.1 }\end{array}$

\section{Imaging and Image Analysis}

Imaging was performed on a Keyence BZ-X810 under 20X magnification. Glp1r was imaged with $647 \mathrm{nM}$ to maximize sensitivity of detection. For tissue sections obtained from C57BL6/J mice, tiled z-stacks of the CeA and surrounding region were captured using optical sectioning. For tissue sections obtained from Glp1r-Cre mice, tiled z-stack images of the 
$\mathrm{CeA}$ and surrounding regions were captured using widefield florescence imaging. Raw images were then stitched and a maximum intensity projection made in Keyence Analyzer. Stitched raw images were obtained for individual channels including DAPI, 647, 550, and $488 \mathrm{~nm}$. Prior to creating composite images, non-specific background signal was removed through image subtraction of images obtained on $488 \mathrm{~nm}$ from those obtained on 647 and $550 \mathrm{~nm}$. No probes were developed using $488 \mathrm{~nm}$ fluorophores. Composite images were then created and processed for quantitative analysis. Cell counts and area measurements were performed in ImageJ (Fiji). Final images were assembled in Adobe Illustrator 25.2.3.

For quantitative analysis of Glp1r expressing neurons across the rostral-caudal axis, three subsections were defined with respect to bregma coordinates as determined using The Mouse Brain in Stereotaxic Coordinates, 4th ed. (Franklin and Paxinos, 2019). The range for anterior, middle, and posterior CeA were defined as follows: the anterior CeA falls within $0.71-1.03 \mathrm{~mm}$ posterior of bregma, middle CeA falls within $1.03-1.43 \mathrm{~mm}$ posterior of bregma, and posterior CeA falls within $1.43-1.79 \mathrm{~mm}$ posterior of bregma. Following acquisition, images were categorized as either anterior, middle, or posterior for quantitative analysis. Classification was determined using anatomical landmarks including the overall size of the CeA, the size and width of the basolateral amygdala, the position of the intercalated cells, and the presence and shape of the stria terminalis. Count data for this analysis was obtained from 6 to 8 images per mouse ( 2 male and 2 female) with 3-4 images of sections $\sim 100 \mu \mathrm{M}$ apart with respect to bregma acquired per hemisphere per mouse.

\section{Patch Clamp Electrophysiology Slice Preparation}

For electrophysiology, animals were removed from their cage and brought to the lab for brain slice preparation. The animal rested in a quiet chamber for $30-45 \mathrm{~min}$ prior to slice preparation to dissipate stress associated with animal transport from the vivarium. Mice were treated with a lethal dose of tribromoethanol (250 mg/kg, i.p.), and, after a deep plane of anesthesia was reached, animals were transcardially perfused with cold, sodium free N-methyl-D-glucamine (NMDG) artificial cerebrospinal fluid (aCSF) [(in mM) $93 \mathrm{~N}$-methyl-D-glucamine, $2.5 \mathrm{KCl}, 1.2$ $\mathrm{NaH}_{2} \mathrm{PO}_{4}, 30 \mathrm{NaHCO}_{3}, 20$ HEPES, 25 Glucose, 5 L-ascorbic acid, 2 Thiourea, 3 sodium pyruvate, $10 \mathrm{MgSO}_{4} \mathrm{X} 7 \mathrm{H}_{2} \mathrm{O}, 0.5 \mathrm{CaCl}_{2}$ $\mathrm{X} 2 \mathrm{H}_{2} \mathrm{O}$ ]. All solutions were saturated with $95 \% \mathrm{CO}_{2}$ and $5 \%$ $\mathrm{O}_{2}$. The brain was rapidly dissected and coronal $300 \mu \mathrm{M}$ sections prepared in ice cold, oxygenated NMDG aCSF using a Leica VT1200S at $0.07 \mathrm{~mm} / \mathrm{s}$. Slices were immediately transferred to $32^{\circ} \mathrm{C}$ NMDG aCSF for $10 \mathrm{~min}$, and then normal $32^{\circ} \mathrm{C}$ aCSF [(in $\mathrm{mM}$ ): $124 \mathrm{NaCl}, 4.4 \mathrm{KCl}, 2 \mathrm{CaCl}_{2}, 1.2 \mathrm{MgSO}_{4}, 1 \mathrm{NaH}_{2} \mathrm{PO}_{4}, 10.0$ glucose, and $26.0 \mathrm{NaHCO}_{3}$ ]. Slices rested in normal aCSF for at least $30 \mathrm{~min}$ prior to recordings.

\section{Recording}

Whole cell patch clamp recordings were performed in the CeA guided by DIC microscopy and mCherry fluorescence. Slices were then transferred to a recording chamber (Warner
Instruments), submerged in normal, oxygenated aCSF and maintained at $32^{\circ} \mathrm{C}$ with a flow rate of $2 \mathrm{ml} / \mathrm{min}$. We patched mCherry-expressing neurons in a balanced fashion in all CeA subdivisions in which they were present. For all recordings we used a potassium gluconate internal solution [(in $\mathrm{mM}): 135$ $\mathrm{C}_{6} \mathrm{H}_{11} \mathrm{KO}_{7}, 5 \mathrm{NaCl}, 2 \mathrm{MgCl}_{2}$, 20 HEPES, 0.6 EGTA, $4 \mathrm{Na}_{2} \mathrm{ATP}$, $0.4 \mathrm{Na}_{2} \mathrm{GTP}$ at a final osmolarity of $290 \mathrm{mOsm}$ at a $\mathrm{pH}$ of 7.3]. For voltage clamp recordings, neurons were voltage clamped at $-70 \mathrm{mV}$ using a Multiclamp $700 \mathrm{~B}$ and currents were digitized with an Axon 1550B digitizer (Molecular Devices, Fremont, CA).

\section{Data Analysis}

Data were analyzed in Clampfit 11.1 (Molecular Devices, San Jose, CA). Membrane capacitance and resistance were determined online using a $-10 \mathrm{mV}$ square pulse. We did not correct for liquid junction potential. Resting membrane potential was determined using a 2' gap free current clamp recording where we took the average following a stabilizing period (usually 30 "). For active neurons, we counted the number of action potentials during this short recording to determine the average spiking frequency. For rheobase, we identified the injected current at the peak of the first action potential. For recordings at $-70 \mathrm{mV}$, we injected a steady variable amount of negative current using the amplifier while recording gap-free in current clamp until the cell reached $-70 \mathrm{mV}$. For determination of spiking subtype, we identified whether the cells had rebound spikes following a negative current injection, the latency to first spike, and if a cell had spike frequency adaptation. Late spiking neurons had a latency to first spike of $>100 \mathrm{~ms}$. Regular spiking neurons had a latency $<100 \mathrm{~ms}$ and minimal spike frequency adaptation. Low threshold bursting neurons had a short latency to the first spike, reduced spike frequency following the first 1-3 action potentials, and had a characteristic rebound spike. For detection of $\mathrm{I}_{\mathrm{h}}$-like currents, we measured the difference in cell current immediately following a negative voltage step to the point of stable current (at least $250 \mathrm{~ms}$ into a $500 \mathrm{~ms}$ voltage step). We measured the difference at each voltage step. To determine the proportion of neurons that demonstrated $\mathrm{I}_{\mathrm{h}}$-like currents we used a threshold of $-20 \mathrm{pA}$ at the most hyperpolarized voltage step $(-120 \mathrm{mV})$. Any cell with $<-20 \mathrm{pA}$ at $-120 \mathrm{mV}$ was empirically categorized as lacking $\mathrm{I}_{\mathrm{h}}$-like current.

\section{Statistics}

All statistical analyses were performed using Graphpad Prism version 9.0.0. For electrophysiology data, original data were analyzed in Clampfit 11.1. Figures were assembled in Adobe Illustrator.

\section{RESULTS}

Previous murine studies have demonstrated the presence of GLP1Rs in the CeA (Cork et al., 2015; Graham et al., 2020); however, we sought to characterize the pattern of GLP-1R expression within a framework of known genetic markers of the CeA. To that end, we used fluorescence in situ hybridization in the CeA to characterize Glp $1 r$ mRNA. In our first experiment, we found that Glp1r-containing cell bodies did not coexpress Prkcd, a member 

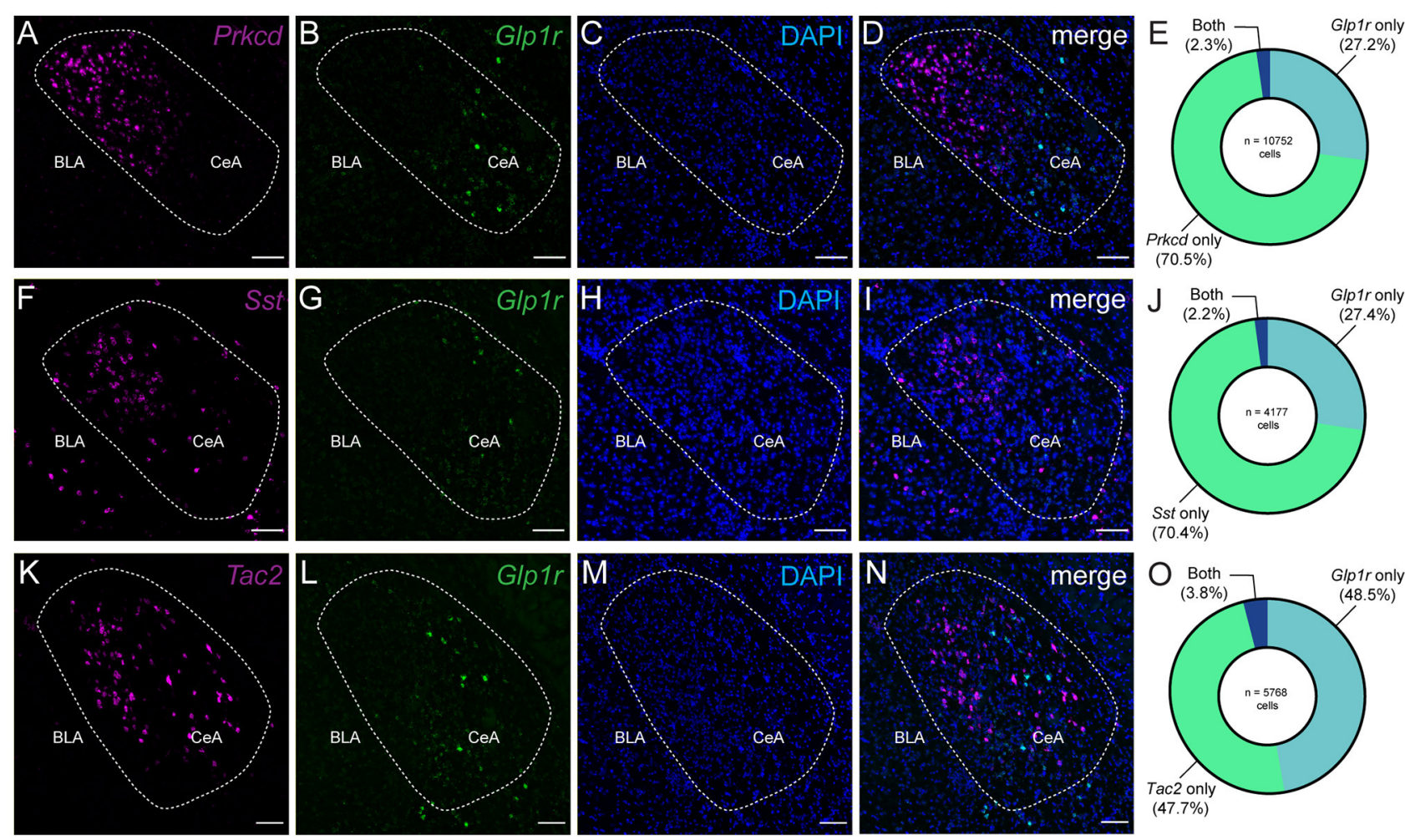

FIGURE 1 | The Glucagon-like peptide 1 receptor is expressed diffusely amongst known genetic markers of the central amygdala. (A-D) Example single channel and merged images of Prkcd, Glp1r, and DAPI mRNA in the CeA of wild type mice. (E) Donut plot depicting percentages of Prkcd, Glp1r, and double-labeled cells. (F-I) Example single channel and merged images of Sst, Glp1r, and DAPI mRNA in the CeA of wild type mice. (J) Donut plot depicting percentages of Sst, Glp1r, and double-labeled cells. (K-N) Example single channel and merged images of Tac2 and Glp1r mRNA, and DAPI in the CeA of wild type mice. (0) Donut plot depicting percentages of Tac2, Glp1r, and double-labeled cells. For (E,J,O), count data were obtained from 6 to 8 images acquired from 4 wildtype C57BL6/J mice (2 male and 2 female) with 3-4 images per hemisphere per mouse. (E) A total of 10,752 cells were counted with 2,688 \pm 558 (s.e.) cells counted per animal. (J) A total of 4,177 cells were counted with 1,044 \pm 82.2 (s.e.) cells counted per animal. (O) A total of 5,788 cells were counted with 1,442 \pm 229 (s.e.) cells counted per animal. Scale bar $=100 \mu \mathrm{m}$ for all images

of the protein kinase $\mathrm{c}$ family that is highly enriched in the lateral and central subdivisions of the CeA (Figures 1A-E) (Haubensak et al., 2010). Of the total neurons counted ( $n=10,752$ cells, 2,688 \pm 558 cells per mouse, 4 mice), only $2.3 \%$ expressed both Prkcd and Glp1r. Notably, the CeL also has an abundant cell group that express the neuropeptide somatostatin (Sst) (Li et al., 2013), and these neurons do not coexpress Prkcd. Together, Sst and Prkcd make up the dominant, nearly exclusive cell types in the CeL (McCullough et al., 2018b). As with Prkcd, we observed only a small population of Glp1r+ neurons coexpress Sst (2.2\%) with respect to the total number of cells counted ( $n=4,177$ cells, $1,044 \pm 82.2$ cells per mouse, 4 mice) (Figures $\mathbf{1 F}-\mathbf{J}$ ). In both experiments, we observed that Glp $1 r$ transcripts were enriched in cell bodies of the medial subnucleus of the CeA (CeM); therefore, we selected Tac2, a neuropeptide whose expression is more enriched in the CeM. Interestingly, Glp1r was not robustly coexpressed with cells that express Tac2 with only $3.8 \%$ of total neurons counted ( $n=5,768$ cells, $1,442 \pm 229$ cells per mouse, 4 mice) showing coexpression (Glp1r+/Tac2+) (Figures 1K-O) (Andero et al., 2014). These data are consistent with a diffuse coexpression pattern of Glp1r within known genetically-defined populations of the CeA.
While amplification-based in situ hybridization is an effective strategy for mRNA detection, we used an alternative cell-labeling approach to quantify the pattern of Glp1r cell distribution in the CeA. In order to validate the Glp1r-Cre mouse line, we employed florescence in situ hybridization techniques to assess the penetrance and fidelity of Cre expression through colocalization analysis of Glp1r and Cre mRNA (Figures 2A-G). Of the Glp1r+ cells counted ( $n=6,595$ cells, $824.4 \pm 125.6$ s.e. cells per mouse), $77.6 \%$ expressed Cre demonstrating high penetrance of this transgenic mouse strain. Additionally, 95.6\% of total Cre + cells counted ( $n=5,351$ cells, $668.9 \pm 101.3$ s.e. cells per mouse) coexpressed Glp1r confirming high fidelity. Together, these data verify the validity of the Glp1r-Cre mouse. Using Glp1r-Cre mice, we stereotaxically injected AAV-DIO-mCherry into the CeA (Figure 2I) which produced an expression pattern of mCherry that resembled native Glp1r mRNA. Using these mice, we serially mounted and counted Glp1r CeA cells across the rostral-caudal axis and observed Glp $1 r^{\mathrm{CeA}}$ cells throughout the entire range of the $\mathrm{CeA}$ and outside, but proximal to the CeA (Figure 2M). In response to this finding, we performed careful cell counts across three equal A-P segments and observed a higher density of Glp $1 r^{\mathrm{CeA}}$ cells in the middle third of the CeA; 

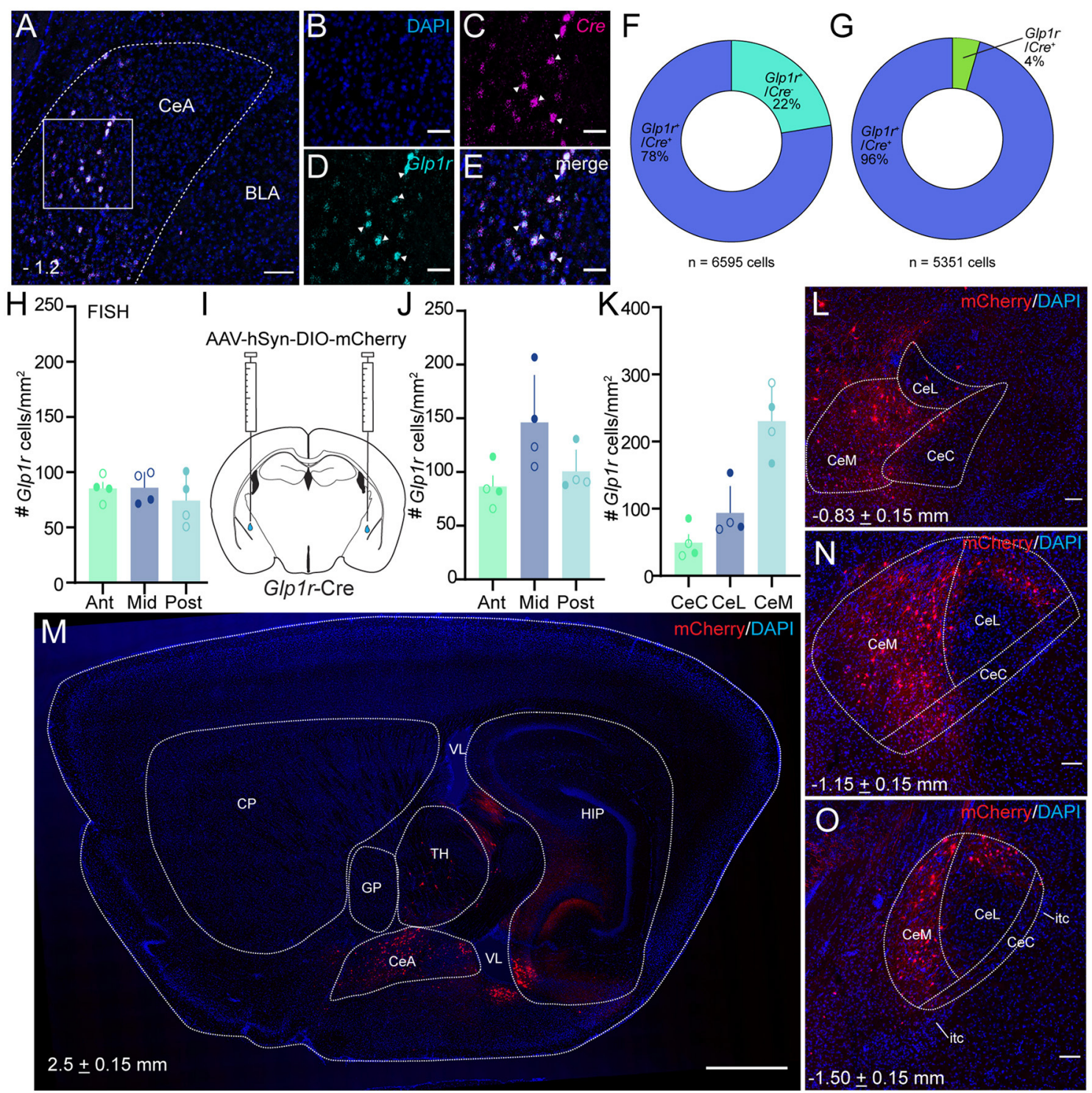

FIGURE 2 | G/p $1 r^{\mathrm{CeA}}$ neurons are distributed across the rostral-caudal axis and enriched in the CeM. (A-G) G/p1r-Cre line validation using dual florescence in situ hybridization. (A) Wide field merged view of CeA with Glp1r in cyan and Cre in magenta. Square represents enlarged, single channel view shown in (B-D) and enlarged merged view in (E). (F) Quantification of Cre mRNA expression within Glp $1 r+$ neurons ( $n=6,595$ cells, $824.4 \pm 125.6$ s.e. cells per mouse). (G) Quantification of Glp1r mRNA expression within Cre+ neurons ( $n=5,351$ cells, $668.9 \pm 101.3$ s.e. cells per mouse). Data shown in (F,G) was obtained from 8 images taken from 4 mice. (H) Schematic of CeA injection of AAV-hSyn-DIO-mCherry in Glp 1r-Cre mice. (I) Quantification of FISH labeled Glp1rCeA neurons across the rostral-caudal axis (counts from 3 to 4 sections per subdivisions per mouse). (J) Quantification of virally tagged G/p 1 r CeA neurons across the rostral-caudal axis $[n=4$, including two male and two female wild type mice; Ordinary one-way ANOVA with Tukey's multiple comparisons test; $f=4.190, p=0.0520$ (Ant vs. Mid), 0.7997 (Ant vs. Post) and 0.1402 (Mid vs. Post)]. (K) Quantification of Glp $1 r^{\mathrm{CeA}}$ neurons at different subdivisions of CeA $[n=4$, including two male and two female wild type mice; Ordinary one-way ANOVA with Tukey's multiple comparisons test; $f=21.89, p=0.3125$ (CeC vs. CeL), 0.0004 (CeC vs. CeM) and 0.0025 (CeL vs. CeM)]. For $\mathbf{( H - K )}$, open circles represent data obtained from female mice and closed circles represent data obtained from male mice. (L,N,O) Example coronal images of Glp $1 r^{\mathrm{CeA}}$ distribution in anterior (L), middle (N) and posterior (O) segments of the CeA. (M) Example sagittal image of Glp1rCeA distribution. Note some viral tagging of putative Glp1r neurons outside the CeA. Scale bars represent $100 \mu \mathrm{m}(\mathbf{A}, \mathbf{M})$ or $50 \mu \mathrm{m}$ (B-E). For all experiments, ${ }^{*} p<0.05,{ }^{* *} p<0.01$, and ${ }^{* * *} p<0.001 . \mathrm{CP}$, caudoputamen; GP, globus pallidus; TH, thalamus; VL, lateral ventricle; HIP, hippocampal region.

however, this did not reach statistical significance (Figure 2J). Similarly, quantification of Glp1r-expressing cells at the mRNA level across the three segments of the CeA revealed no difference in the density of Glp1r-expressing cells (Figure $2 \mathbf{H}$ ). We then used anatomical landmarks and atlases to count Glp1r+ cells in the lateral, capsular, and medial subdivisions of the CeA and identified a significant enrichment in the density of $G l p 1 r^{\mathrm{CeA}}$ cells in the CeM (Figures $\mathbf{2 K}, \mathbf{L}, \mathbf{N}, \mathbf{O}$ ). Thus, Glp $1 r^{\mathrm{CeA}}$ cells are expressed throughout the rostral-caudal axis of the CeA and enriched in the output subnucleus of the CeA - the CeM. 
To gain insights into the functional nature of $G l p 1 r^{\mathrm{CeA}}$ cells, we used ex vivo brain slices combined with whole cell patch clamp electrophysiology. We injected male and female Glp1rCre mice with AAV-DIO-mCherry and waited 3-4 weeks prior to preparing fresh brain slices (Figures 3A,B). Glp $1 r^{\mathrm{CeA}}$ neurons were identified using DIC and epifluorescence (Figure 3C). On average, Glp $1 r^{\mathrm{CeA}}$ neurons had a membrane capacitance $(\mathrm{Cm})$ of $45.75 \pm 3.03 \mathrm{pF}$ and a membrane resistance $(\mathrm{Rm})$ of $309 \pm 26.9 \mathrm{~m} \Omega$ (Figures 3E,F). In gap free current clamp mode, we measured the average resting membrane potential (RMP) and excitability of Glp1r ${ }^{\mathrm{CeA}}$ neurons. Interestingly, we observed that $42 \%$ of Glp $1 r^{\mathrm{CeA}}$ neurons are spontaneously active at rest (Figure 3D) and had an average RMP of $-51.93 \pm$ $1.59 \mathrm{mV}$ (Figure 3G). Spontaneously active Glp1r ${ }^{\mathrm{CeA}}$ neurons displayed an average firing frequency of $0.47 \mathrm{~Hz}$. To understand if the activity state of $G l p 1 r^{\mathrm{CeA}}$ neurons is associated with neurophysiological characteristics, we analyzed the $\mathrm{Cm}, \mathrm{Rm}$, and RMP within both active and inactive Glp1r CeA neuron subgroups. Consistent with their activity states, active neurons had a higher RMP than inactive neurons (active $=-43.48 \pm$ $1.217 \mathrm{mV}$ vs. inactive $=-57.97 \pm 1.878 \mathrm{mV}$ ), but we observed no differences in $\mathrm{Cm}$ (active $=40.66 \pm 5.039 \mathrm{pF}$ vs. inactive $=49.39 \pm 3.681 \mathrm{pF}$ ) or $\mathrm{Rm}$ (active $=360.5 \pm 53.98 \mathrm{~m} \Omega$ vs. inactive $=272.3 \pm 23.99 \mathrm{~m} \Omega$ ) (Figures 3E-G). We also quantified differences in basal neuronal properties in male vs. female $G l p 1 r^{\mathrm{CeA}}$ neuron subgroups. Surprisingly, we observed a sex difference in the $\mathrm{Cm}$ of $G l p 1 r^{\mathrm{CeA}}$ neurons, where Glp $1 r^{\mathrm{CeA}}$ neurons from female mice $(39.56 \pm 4.584 \mathrm{pF})$ had a significantly lower $\mathrm{Cm}$ than male from male mice $(51.95 \pm 3.646 \mathrm{pF})$ (Figure 3E). We observed no statistically significant differences in the $\mathrm{Rm}$ (male $=260.1 \pm 26.51 \mathrm{~m} \Omega$ vs. female $=358 \pm 45.25$ $\mathrm{m} \Omega$ ) or RMP (male $=-53.53 \pm 2.691 \mathrm{mV}$ vs. female $=-50.34$ $\pm 1.679 \mathrm{mV}$ ) between male and female mice (Figures 3E,G).

Using whole cell patch clamp electrophysiology, we probed other neurophysiological phenomena that typify CeA neurons. On average, Glp1r ${ }^{\mathrm{CeA}}$ neurons show $\mathrm{I}_{\mathrm{h}}$-like currents (Figures 3K,L,Q) though we observed that $20 \%$ of Glp $1 r^{\mathrm{CeA}}$ neurons showed no detectable $\mathrm{I}_{\mathrm{h}}$-like currents (inset in Figure 3K). We quantified $\mathrm{I}_{\mathrm{h}}$-like currents in both active and inactive neurons and observed a statistically significant reduction in $\mathrm{I}_{\mathrm{h}}$-like currents in active neurons that was most evident at a hyperpolarized potential of $-120 \mathrm{mV}$ (Figure 3K). Similarly, we observed a significant reduction in $\mathrm{I}_{\mathrm{h}}$-like current in $G l p 1 r^{\mathrm{CeA}}$ neurons from female mice relative to male mice that was evident at multiple holding potentials (Figure 3L).

In response to ramping positive current injections in current clamp mode, Glp $1 r^{\mathrm{CeA}}$ neurons demonstrated action potentials with an average threshold of $-32.8 \pm 0.800 \mathrm{mV}$ (Figure $3 \mathbf{H}$ ). We observed no statistical differences between active $(-33.08 \pm$ $1.292 \mathrm{mV})$ and inactive $(-32.64 \pm 1.034 \mathrm{mV})$ or between male $(-32.77 \pm 1.108 \mathrm{mV})$ and female $(-32.88 \pm 1.179 \mathrm{mV})$ Glp $1 r^{\mathrm{CeA}}$ neurons. As nearly half of $G l p 1 r^{\mathrm{CeA}}$ neurons were spontaneously active, we measured the current needed to produce an action potential (rheobase) at rest and while holding the cell at $-70 \mathrm{mV}$. At rest, the rheobase for Glp1r ${ }^{\mathrm{CeA}}$ neurons was $64.26 \pm$ $6.45 \mathrm{pA}$ whereas at $-70 \mathrm{mV}$ the rheobase was $118.8 \pm 10.74 \mathrm{pA}$ (Figures 3I,J). At rest, we found that active neurons (39.22 $\pm 8.305 \mathrm{pA})$ demonstrate a significantly lower rheobase than inactive neurons $(82.15 \pm 7.814 \mathrm{pA})$ (Figure 3I). Interestingly, active neurons $(76.99 \pm 9.579 \mathrm{pA})$ displayed a significantly lower rheobase than inactive neurons $(140.9 \pm 16.24 \mathrm{pA})$ even when held at $-70 \mathrm{mV}$ (Figure 3J). We observed no statistically significant differences in Glp1r ${ }^{\mathrm{CeA}}$ neuron rheobase between male and female mice at rest (males: $69.97 \pm 8.487 \mathrm{pA}$ vs. females: $58.56 \pm 9.756 \mathrm{pA}$ ) (Figure 3I) or at $-70 \mathrm{mV}$ (males: $118.3 \pm$ $14.66 \mathrm{pA}$ vs. females: $115.6 \pm 18.79 \mathrm{pA}$ ) (Figure 3J).

To further characterize Glp $1 r^{\mathrm{CeA}}$ neuronal excitability, we measured changes in membrane voltage and action potentials in response to increasingly positive square current steps in Glp $1 r^{\mathrm{CeA}}$ neurons held at their resting membrane potential and at $-70 \mathrm{mV}$. In response to current injections, we found that active $G l p 1 r^{\mathrm{CeA}}$ neurons display significantly more action potentials than inactive $G l p 1 r^{\mathrm{CeA}}$ neurons at rest (Figure $3 \mathbf{M}$ ) or at $-70 \mathrm{mV}$ (Figure 3O) and the increase in the number of action potentials was most pronounced at the highest positive current injections. We observed no difference in current injection-induced action potential firing between male and female Glp $1 r^{\mathrm{CeA}}$ neurons either at rest (Figure 3N) or held at $-70 \mathrm{mV}$ (Figure 3P). Forty-one percent of Glp1r ${ }^{\mathrm{CeA}}$ neurons showed spike frequency adaptation at depolarizing current steps (data not shown). Using these data we also classified each neuron according to spike characteristics (Dumont et al., 2002; Chieng et al., 2006; Herman et al., 2013; $\mathrm{Li}$ and Sheets, 2018). The majority (73\%) of Glp1r ${ }^{\mathrm{CeA}}$ neurons were a low threshold bursting (LTB) subtype with a characteristic rebound action potential that occurs following cessation of a hyperpolarizing current step and low latency action potentials in response to depolarizing current steps (Figure 3R). LTB Glp $1 r^{\mathrm{CeA}}$ neurons were equally distributed between active and inactive neurons (inset pie chart in Figure 3R). Moreover, we did not observe a significant difference in the overall distribution of spike types amongst active/inactive or male/female $G l p 1 r^{\mathrm{CeA}}$ neurons (Figure 3S). The sites of these recordings are presented in Figure 3T.

\section{DISCUSSION}

In this study, we performed the first deep anatomical characterization of Glp1r expression and neurophysiology study of Glp1r-expressing cells in the CeA of C57BL6/J mice. At an anatomical level, a previous study using a Glp1r-Cre mouse strain identified Glp1r-expressing cells throughout the brain including the CeA (Cork et al., 2015). Their observations are mirrored by a study from Jensen and colleagues who used a novel GLP-1R antibody and observed GLP-1R protein expression in the CeA (Jensen et al., 2018). In a recent study using a transgenic mouse with an mApple-tagged GLP-1R, the authors rigorously mapped sites of GLP-1R expression throughout the brain and made similar observations, including robust expression in the CeA (Graham et al., 2020). Consistent with their findings using a transgenic mouse, the authors used fluorescence in situ hybridization as a convergent method to characterize native Glp1r mRNA. They showed that Glp1r mRNA is present in the CeA and medial amygdala, and, in the CeA, Glp1r is coexpressed 


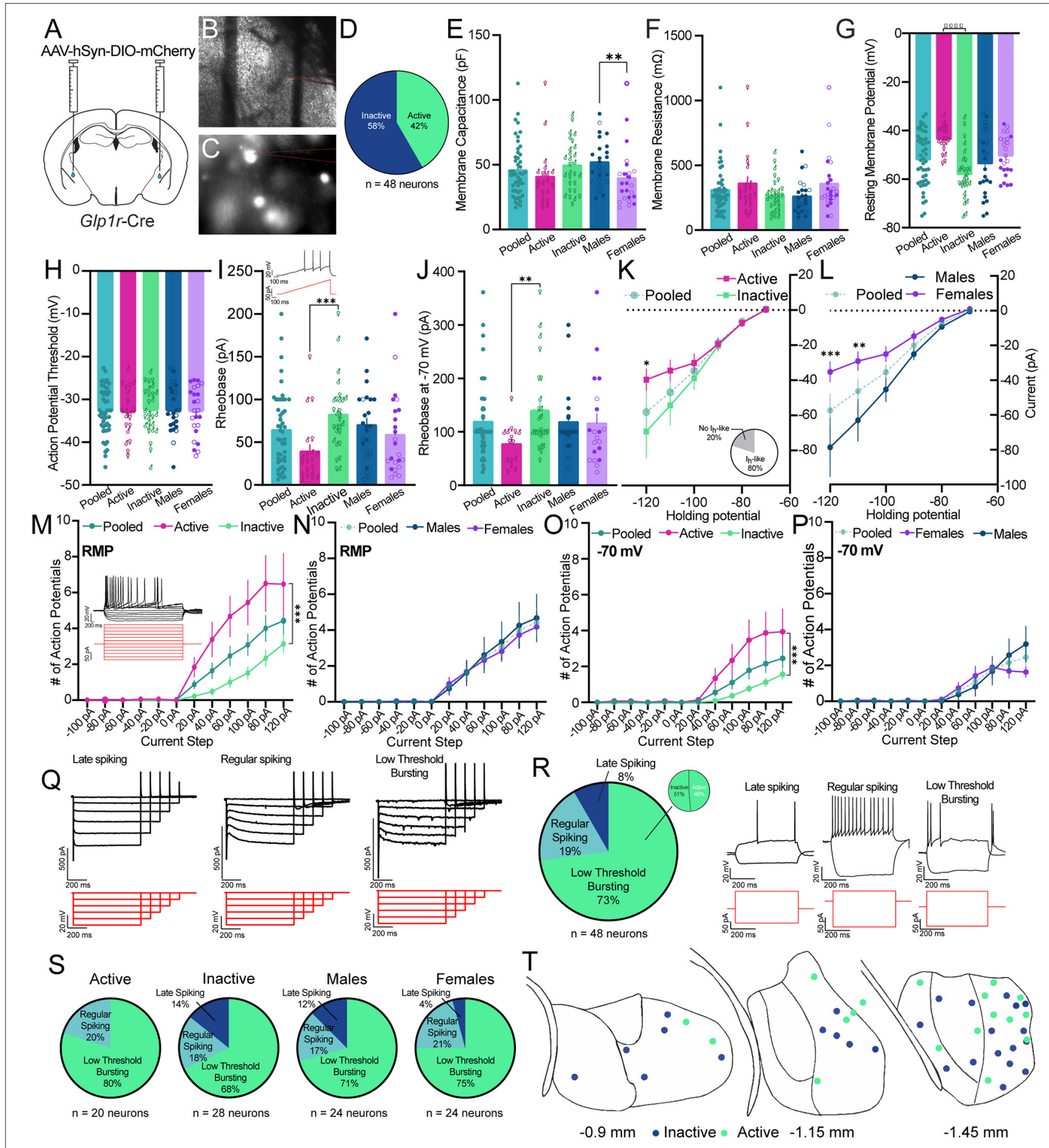

FIGURE 3 | Ex vivo patch clamp electrophysiological characterization of Glp $1 r^{\mathrm{CeA}}$ neurons. (A) Schematic of CeA injection of pAAV-hSyn-DIO-mCherry in Glp1r-Cre mice. (B,C) Example images of live brain slices and fluorescently labeled cells in the CeA during electrophysiological recordings from G/p $1 r$-Cre mice. (D) Pie chart illustrating the number of G/p $1 r^{\mathrm{CeA}}$ neurons that were active/inactive. (E) Average membrane capacitance $(\mathrm{Cm})$ of G/p $1 r^{\mathrm{CeA}}$ neurons in all cells (pooled) (teal), in neurons empirically determined to be active (pink) or inactive (green), or in neurons recorded from male (blue) or female (purple) mice. Within the active/inactive data sets the sex of the mouse from which the neuron was recorded is depicted by use of male and female symbols. Within the male/female data sets the activity state of the neuron is indicated by a closed (inactive) or open (active) symbol. Data were analyzed using a Mann-Whitney $U$ test between active/inactive $(p=0.0736)$ and male/female $(p=0.0063)$ subgroups. (F) Average membrane resistance $(\mathrm{Rm})$ of $\mathrm{G} / \mathrm{p} 1 \mathrm{r}^{\mathrm{CeA}}$ neurons in all cells (pooled) or active, inactive, male, or female subgroups. The symbol conventions used are identical to those used in $(\mathbf{E})$. Data were analyzed using a Mann-Whitney $U$ test between active/inactive $(p=0.2296)$ and 
FIGURE 3 | male/female ( $p=0.0609$ ) subgroups. (G) Average resting membrane potential (Vrest) of all G/p1 ${ }^{\mathrm{CeA}}$ neurons (pooled), or active, inactive, male, or female subgroups. The symbol conventions used are identical to those used in (E). Data were analyzed using a Mann Whitney $U$ test between active/inactive $(p<0.0001)$ and male/female $(p=0.4677)$ subgroups. $(\mathbf{H})$ Average action potential threshold of all Glp $1 r^{\mathrm{CeA}}$ neurons (pooled), or active, inactive, male, or female subgroups. The symbol conventions used are identical to those used in (E). Data were analyzed using a Mann-Whitney $U$ test between active/inactive $(p=0.8319)$ and male/female $(p$ $=0.9796$ ) subgroups. (I) Average rheobase of all G/p $1 r^{\mathrm{CeA}}$ neurons (pooled), or active, inactive, male, or female subgroups at rest. The symbol conventions used are identical to those used in (E). Data were analyzed using a Mann-Whitney $U$ test between active/inactive $(p=0.0001)$ and male/female $(p=0.2276)$ subgroups. Inset: representative cell response in current clamp mode to gradually increasing depolarizing current injection (rheobase). Bottom: Stimulation waveform (J) Average rheobase current of all Glp $1 r^{\mathrm{CeA}}$ neurons (pooled), or active, inactive, male, or female subgroups at $-70 \mathrm{mV}$. The symbol conventions used are identical to those used in (E). Data were analyzed using a Mann-Whitney $U$ test between active/inactive $(p=0.0058)$ and male/female $(p=0.4877)$ subgroups. (K) Average $I_{h}$-like current at hyperpolarizing membrane potentials in all, active, or inactive G/p1 $r^{\mathrm{CeA}}$ neurons. Active and inactive subgroups were compared using 2-way repeated measures ANOVA with Sidak's multiple comparisons test at each holding potential; Holding potential X Activity state: $F_{(5,215)}=2.413, p=0.0373$; Holding potential: $F_{(5,215)}=$ 28.95, $p<0.0001$; Activity state: $F_{(1,43)}=1.467, p=0.2324$. The inset pie chart displays the proportion of neurons that demonstrate significant $I_{h}$-like current using a threshold of $-20 \mathrm{pA}$ for the -120 - $\mathrm{mV}$ voltage step. (L) Average $\mathrm{I}_{\mathrm{h}}$-like current at hyperpolarizing membrane potentials in all, male, or female G/p $1 \mathrm{r}^{\mathrm{C} e A}$ neurons. Male and female subgroups were compared using 2-way repeated measures ANOVA with Sidak's multiple comparisons test at each holding potential; Holding potential $X$ Sex: $F_{(5,215)}=4.976, p=0.0002$; Holding potential: $F_{(5,215)}=34.80, p<0.0001$; Sex: $F_{(1,43)}=6.394, p=0.0152$. (M) Action potentials in response to depolarizing current steps at rest in all, active, or inactive G/p1r $r^{\mathrm{CeA}}$ neurons. Active and inactive subgroups were compared using 2-way repeated measures ANOVA with Sidak's multiple comparisons test at each current injection; Current Injected X Activity state: $F_{(11,473)}=7.881, p<0.0001$; Current Injected: $F_{(11,473)}=33.93, p<0.0001$; Activity state: $F_{(1,43)}=11.83, p=0.0013$. Top: representative cell response in current clamp mode to increasing current steps. (N) Action potentials in response to depolarizing current steps at rest in all, male, or female G/p1r CeA neurons. Male and female subgroups were compared using 2-way repeated measures ANOVA with Sidak's multiple comparisons test at each current injection; Current Injected X Sex: $F_{(11,473)}=0.2256, p=0.9958$; Current Injected: $F_{(11,473)}=24.96, p<0.0001$; Sex: $F_{(1,43)}=0.07738, p=0.7822$. (0) Action potentials in response to depolarizing current steps at $-70 \mathrm{mV}$ in all, active, or inactive G/p1 $1 r^{\mathrm{CeA}}$ neurons. Active and inactive subgroups were compared using 2-way repeated measures ANOVA with Sidak's multiple comparisons test at each current injection; Current Injected $X$ Activity state: $F_{(11,418)}=5.822, p<0.0001$; Current Injected: $F_{(11,473)}=19.79, p<0.0001$; Activity state: $F_{(1,38)}=8.353, p=0.0063$. (P) Action potentials in response to depolarizing current steps at $-70 \mathrm{mV}$ in all, male, or female G/p $1 r^{\mathrm{CeA}}$ neurons. Male and female subgroups were compared using 2-way repeated measures ANOVA with Sidak's multiple comparisons test at each current injection; Current Injected X Sex: $F_{(11,418)}=1.358, p=0.1903 ;$ Current Injected: $F_{(11,418)}=$ 14.02, $p<0.0001$; Sex: $F_{(1,38)}=0.05488, p=0.8160$. (Q) Example $I_{h}$-like currents across three observed spiking subtypes for G/p $1 r^{\text {CeA }}$ neurons. The capacitive currents have been cropped for visualization purposes. Voltage step waveforms are shown below each cell. (R) Pie chart illustrating the distribution of spiking subtypes (late spiking, regular spiking, or low-threshold bursting) for Glp1 $r^{\mathrm{CeA}}$ neurons in response to hyperpolarizing or depolarizing current steps. Example late spiking, regular spiking and low-threshold bursting G/p1r CeA neurons in response to depolarizing current steps are shown to the right. (S) Pie charts illustrating the distribution of spiking subtypes among inactive, active, male, or female subgroups. The distributions of active/inactive and male/female subgroups were compared using a Chi-squared test for trend $\left(p=0.1511\right.$ for active/inactive and $p=0.4911$ for male/female). (T) Schematic of distribution of recorded Glp $1 r^{\text {CeA }}$ neurons. Blue dots represent inactive and green dots represent active neurons. $N=48$ neurons from male $(n=10)$ and female $(n=7)$ mice with 24 neurons per sex.

with the GABAergic neuronal biosynthetic enzyme Gad1. Since Gad1 is only expressed in neurons, we infer that the Glp1r cells we characterized in our study are primarily neuronal.

We observed that Glp1r ${ }^{\mathrm{CeA}}$ neurons are not defined by the sole coexpression with either Prkcd, Sst, or Tac2. Prkcd is a gene that marks a population of lateral and capsular CeA neurons that are activated by aversive unconditioned stimuli, inhibited by aversive conditioned stimuli, and inhibit food intake when activated (Haubensak et al., 2010; Cai et al., 2014; Cui et al., 2017; McCullough et al., 2018a). Sst is a neuropeptidergic gene that marks a population of lateral and medial CeA neurons almost completely distinct from Prkcd that are activated by aversive conditioned stimuli, modulate passive and active defensive responses, and promote freezing and behavioral cessation when activated using optogenetics (Li et al., 2013; Yu et al., 2016). Tac2 is a neuropeptidergic gene enriched in medial and some lateral CeA neurons and modulates the expression of conditioned fear (Andero et al., 2014, 2016). A recent study identified that $\sim 50 \%$ of Glp1r ${ }^{\mathrm{CeA}}$ neurons coexpress the cytokine interleukin-6 (IL-6) and that direct administration of IL- 6 to the CeA could produce a subtle decrease in feeding, but the functional impact of IL-6 in combination with CeA GLP-1Rs is unclear (Anesten et al., 2019). The limited overlap we observed between Prkcd/Sst/Tac2 and Glp1r in the CeA suggests that the anorexigenic actions of peripherally-applied or intracerebral GLP-1R agonists are likely not due to the direct activation of these neurons, but do not preclude their activation through indirect network effects (Gabery et al., 2020). Further experiments using in vivo recordings are necessary to test direct and indirect models of action of GLP-1R agonists on these genetically defined CeA neurons.

Using Cre-dependent viral tracing in combination with Glp1rCre mice, we performed cell counts of Glp1r cells across the rostral-caudal axis and in CeA subnuclei. Glp1r ${ }^{\mathrm{CeA}}$ neurons are located throughout the rostral-caudal axis with a non-significant increase in the middle third of the CeA and are more densely distributed in the CeM. Coincidently, the CeA also receives innervation from Preproglucagon (PPG, the precursor gene of GLP-1)-expressing neurons in the nucleus of the solitary tract and this innervation is most dense in the CeM (LlewellynSmith et al., 2011; Williams et al., 2018; Anesten et al., 2019). The functional and behavioral role of PPG inputs to the CeA are unknown.

Using brain slice electrophysiology, we performed a neurophysiological characterization of $G l p 1 r^{\mathrm{CeA}}$ neurons in male and female mice. Surprisingly, we found that nearly half of these neurons are active at rest. We analyzed neurophysiological characteristics associated with inherent neuronal activity states. Not surprisingly, we found that active Glp1r ${ }^{\mathrm{CeA}}$ neurons have a higher resting membrane potential than inactive neurons and are more sensitive to current injections. Interestingly, the increased sensitivity of active Glp1r $\mathrm{CeA}^{\mathrm{CA}}$ neurons to current injectioninduced action potentials persists even when held at an identical polarized membrane potential of $-70 \mathrm{mV}$. As seen in other CeA neuronal physiology studies, Glp $1 r^{\mathrm{CeA}}$ neurons on average show hyperpolarization-activated $\left(\mathrm{I}_{\mathrm{h}}\right)$ currents; however, we did 
observe some cells for which no substantial $\mathrm{I}_{\mathrm{h}}$-like current was detected $(20 \%)$. Interestingly, we found reduced $\mathrm{I}_{\mathrm{h}}$-like currents in $G l p 1 r^{\mathrm{CeA}}$ neurons recorded from female mice and that, on average, these neurons have a lower membrane capacitance. The functional implications of these sex differences is unclear although they may be related to overall morphometric size differences that have been reported in the amygdala (Hines et al., 1992; Qiu et al., 2018). For Glp1r ${ }^{\mathrm{CeA}}$ neurons, we characterized their excitability using current injections in current clamp mode and determined their spiking subtype. Overall, we found that $G l p 1 r^{\mathrm{CeA}}$ neurons are predominantly a low threshold bursting subtype characterized by rebound spikes from hyperpolarizing current injections. We speculate that cells of the low threshold burst subtype may be particularly sensitive to excitatory input or activation of intracellular signaling pathways that enhance excitability like GLP-1Rs. Several heroic neurophysiological studies of CeA neurons in general or CeL neurons demonstrate a diversity of spiking types (Dumont et al., 2002; Chieng et al., 2006; Hunt et al., 2017; Adke et al., 2021). Only a couple of studies have focused neurophysiological recordings on the $\mathrm{CeM}$, which is more diverse with respect to gene expression and axonal projections (Herman et al., 2013; Li and Sheets, 2018; McCullough et al., 2018b). Interestingly Li and colleagues showed that periaqueductal gray (PAG)-projecting CeA neurons are distributed in the CeL and CeM and did an extensive study of their firing properties and excitability broken down by firing subtype. PAG-projecting CeM neurons are heterogenous and comprised of equal proportions of regular spiking, fast-spiking, and bursting subtype and demonstrate voltage sag in response to hyperpolarizing current injections indicative of $\mathrm{I}_{\mathrm{h}}$-like currents (Li and Sheets, 2018). However, the authors did not observe sex differences in the voltage sag of PAG-projecting $\mathrm{CeM}$ neurons. These data suggest that $G l p 1 r^{\mathrm{CeA}}$ neurons are likely distinct from CeM-projecting neurons, however further studies using anterograde and tracers are needed to rule out this possibility. CeA neurons that express corticotropin releasing factor receptor 1 (CRFR1 or Crhrl) are distributed in the medial and lateral subdivisions of the $\mathrm{CeA}$, and the reported membrane properties, resting membrane potential, and spiking types most closely match those in this study (Herman et al., 2013). CRFR1, like GLP-1R, is a $\mathrm{G}_{\alpha s}$-coupled receptor so it will be interesting to know in the future if $G l p 1 r^{\mathrm{CeA}}$ neurons coexpress Crhrl.

GLP-1R agonists are widely prescribed for the treatment of type II diabetes and they also have the added benefit

\section{REFERENCES}

Adams, J. M., Pei, H., Sandoval, D. A., Seeley, R. J., Chang, R. B., Liberles, S. D., et al. (2018). Liraglutide modulates appetite and body weight through glucagon-like peptide 1 receptor-expressing glutamatergic neurons. Diabetes 67, 1538-1548. doi: $10.2337 / \mathrm{db} 17-1385$

Adke, A. P., Khan, A., Ahn, H., Becker, J. J., Wilson, T. D., Valdivia, S., et al. (2021). Cell-type specificity of neuronal excitability and morphology in the central amygdala. eNeuro 8, 1-28. doi: 10.1523/ENEURO.040220.2020 of suppressing appetite and producing weight loss (Drucker, 2018). Recently the FDA approved the use of semaglutide (a new generation GLP-1R agonist) for the treatment of obesity. Unfortunately, the mechanisms by which these drugs exert their anorexigenic effects in the brain is still unclear. We hypothesize that the CeA, in addition to the hypothalamus and hindbrain, is an important limbic nucleus that mediates some of the anorexigenic effects of these drugs. Because the CeA is a nucleus that receives endogenous GLP-1 through preproglucagon inputs from the NTS, we also speculate that that CeA GLP-1Rs may play a role in feeding, emotional, and motivational processing. One study using non-human primates demonstrated that GLP-1Rs are present in the amygdala (Heppner et al., 2015), but further research using post-mortem tissue from humans is needed to demonstrate that GLP-1Rs are expressed in the human amygdala. In addition, basic research using genetic and neural circuit approaches are needed to characterize a circuit logic for the mode of action of GLP-1R agonists.

\section{DATA AVAILABILITY STATEMENT}

The raw data supporting the conclusions of this article will be made available by the authors without undue reservation.

\section{ETHICS STATEMENT}

The animal study was reviewed and approved by UAB IACUC.

\section{AUTHOR CONTRIBUTIONS}

NZ, EC, CL, SK, and MD collected data for the manuscript. NZ and $\mathrm{JH}$ analyzed the data. $\mathrm{NZ}, \mathrm{SV}$, and $\mathrm{JH}$ wrote the manuscript and assembled the figures. JH designed the study. All authors contributed to the article and approved the submitted version.

\section{FUNDING}

This project was supported by K01DK115902 and R03DK129561 to $\mathrm{JH}$ and the UAB Nutrition Obesity Research Center Pilot and Feasibility Program P30DK056336.

\section{ACKNOWLEDGMENTS}

We would like to thank Stephen Liberles and Michael Krashes for providing the Glp1r-Cre mice. 
consolidation. Neuron 83, 444-454. doi: 10.1016/j.neuron.2014. 05.028

Anesten, F., Dalmau Gasull, A., Richard, J. E., Farkas, I., Mishra, D., Taing, L., et al. (2019). Interleukin-6 in the central amygdala is bioactive and colocalised with glucagon-like peptide-1 receptor. J. Neuroendocrinol. 31:e12722. doi: 10.1111/jne.12722

Burmeister, M. A., Ayala, J., Drucker, D. J., and Ayala, J. E. (2013). Central glucagon-like peptide 1 receptor-induced anorexia requires glucose metabolism-mediated suppression of AMPK and is impaired by central fructose. Am. J. Physiol. Endocrinol. Metab. 304, 677-685. doi: 10.1152/ajpendo.00446.2012

Burmeister, M. A., Ayala, J. E., Smouse, H., Landivar-Rocha, A., Brown, J. D., Drucker, D. J., et al. (2017). The hypothalamic glucagon-like peptide 1 receptor is sufficient but not necessary for the regulation of energy balance and glucose homeostasis in mice. Diabetes 66, 372-384. doi: 10.2337/db16-1102

Cai, H., Haubensak, W., Anthony, T. E., and Anderson, D. J. (2014). Central amygdala PKC- $\delta+$ neurons mediate the influence of multiple anorexigenic signals. Nat. Neurosci. 17, 1240-1248. doi: 10.1038/nn.3767

Chieng, B. C. H., Christie, M. J., and Osborne, P. B. (2006). Characterization of neurons in the rat central nucleus of the amygdala: cellular physiology, morphology, and opioid sensitivity. J. Comp. Neurol. 927, 910-927. doi: $10.1002 /$ cne. 21025

Cork, S. C., Richards, J. E., Holt, M. K., Gribble, F. M., Reimann, F., and Trapp, S. (2015). Distribution and characterisation of Glucagon-like peptide1 receptor expressing cells in the mouse brain. Mol. Metab. 4, 718-731. doi: 10.1016/j.molmet.2015.07.008

Cui, Y., Lv, G., Jin, S., Peng, J., Yuan, J., He, X., et al. (2017). Article A central amygdala-substantia innominata neural circuitry encodes aversive reinforcement signals article A central amygdala-substantia innominata neural circuitry encodes aversive reinforcement signals. Cell Rep. 21, 1770-1782. doi: 10.1016/j.celrep.2017.10.062

Douglass, A. M., Kucukdereli, H., Ponserre, M., Markovic, M., Gründemann, J., Strobel, C., et al. (2017). Central amygdala circuits modulate food consumption through a positive-valence mechanism. Nat. Neurosci. 20, 1384-1394. doi: 10.1038/nn.4623

Drucker, D. J. (2018). Mechanisms of action and therapeutic application of glucagon-like peptide-1. Cell Metab. 27, 740-756. doi: 10.1016/j.cmet.2018.03.001

Dumont, É. C., Martina, M., Samson, R. D., Drolet, G., and Paré, D. (2002). Physiological properties of central amygdala neurons: species differences. Eur. J. Neurosci. 15, 545-552. doi: 10.1046/j.0953-816x.2001.01879.x

Franklin, K., and Paxinos, G. (2019). Paxinos and Franklin's the Mouse Brain in Stereotaxic Coordinates, Compact. San Diego, CA: Elsevier, Inc.

Gabery, S., Secher, A., Knudsen, L. B., Gabery, S., Salinas, C. G., Paulsen, S. J., et al. (2020). Semaglutide lowers body weight in rodents via distributed neural pathways Semaglutide lowers body weight in rodents via distributed neural pathways. JCI Insight 5:e133429. doi: 10.1172/jci.insight.133429

Graham, D. L., Durai, H. H., Trammell, T. S., Noble, B. L., Mortlock, D. P., Galli, A., et al. (2020). A novel mouse model of glucagon-like peptide-1 receptor expression: a look at the brain. J. Comp. Neurol. 528, 2445-2470. doi: $10.1002 / \mathrm{cne} .24905$

Hardaway, J. A., Halladay, L. R., Mazzone, C. M., Pati, D., Bloodgood, D. W., Kim, M., et al. (2019). Central amygdala prepronociceptin-expressing neurons mediate palatable food consumption and reward. Neuron. 102, 1037-1052.e7. doi: 10.1016/j.neuron.2019.03.037

Haubensak, W., Kunwar, P. S., Cai, H., Ciocchi, S., Wall, N. R., Ponnusamy, R., et al. (2010). Genetic dissection of an amygdala microcircuit that gates conditioned fear. Nature 468, 270-276. doi: 10.1038/nature09553

Hayes, M. R., Bradley, L., and Grill, H. J. (2009). Endogenous hindbrain glucagon-like peptide- 1 receptor activation contributes to the control of food intake by mediating gastric satiation signaling. Endocrinology 150, 2654-2659. doi: 10.1210/en.2008-1479

Heppner, K. M., Kirigiti, M., Secher, A., Paulsen, S. J., Buckingham, R., Pyke, C., et al. (2015). Expression and distribution of glucagon-like peptide-1 receptor mRNA, protein and binding in the male nonhuman primate (Macaca mulatta) Brain. Endocrinology 156, 255-267. doi: 10.1210/en.2014-1675

Herman, M. A., Contet, C., Justice, N. J., Vale, W., and Roberto, M. (2013). Novel subunit-specific tonic GABA currents and differential effects of ethanol in the central amygdala of CRF receptor-1 reporter mice. J. Neurosci. 33, 3284-3298. doi: 10.1523/JNEUROSCI.2490-12.2013

Hines, M., Allen, L. S., and Gorski, R. A. (1992). Sex differences in subregions of the medial nucleus of the amygdala and the bed nucleus of the stria terminalis of the rat. Brain Res. 579, 321-326. doi: 10.1016/0006-8993(92)90068-K

Hunt, S., Sun, Y., Kucukdereli, H., Klein, R., and Sah, P. (2017). Intrinsic circuits in the lateral central amygdala. eNeuro 4, 1-18. doi: 10.1523/ENEURO.0367-16.2017

Ip, C. K., Zhang, L., Farzi, A., Qi, Y., Clarke, I., Reed, F., et al. (2019). Amygdala NPY circuits promote the development of accelerated obesity under chronic stress conditions. Cell Metab. 30, 111-128.e6. doi: 10.1016/j.cmet.2019.04.001

Janak, P. H., and Tye, K. M. (2015). From circuits to behaviour in the amygdala. Nature 517, 284-292. doi: 10.1038/nature14188

Jensen, C. B., Pyke, C., Rasch, M. G., Dahl, A. B., Knudsen, L. B., and Secher, A. (2018). Characterization of the glucagonlike peptide-1 receptor in male mouse brain using a novel antibody and in situ hybridization. Endocrinology 159, 665-675. doi: 10.1210/en.2017-00812

Kanoski, S. E., Fortin, S. M., Arnold, M., Grill, H. J., and Hayes, M. R. (2011). Peripheral and central GLP-1 receptor populations mediate the anorectic effects of peripherally administered GLP-1 receptor agonists, liraglutide and exendin-4. Endocrinology 152, 3103-3112. doi: 10.1210/en.201 1-0174

Li, H., Penzo, M. A., Taniguchi, H., Kopec, C. D., Huang, Z. J., and Li, B. (2013). Experience-dependent modification of a central amygdala fear circuit. Nat. Neurosci. 16, 332-339. doi: 10.1038/ nn. 3322

Li, J., and Sheets, P. L. (2018). The central amygdala to periaqueductal gray pathway comprises intrinsically distinct neurons differentially affected in a model of inflammatory pain. J. Physiol. 24, 6289-6305. doi: 10.1113/JP2 76935

Llewellyn-Smith, I. J., Reimann, F., Gribble, F. M., and Trapp, S. (2011) Preproglucagon neurons project widely to autonomic control areas in the mouse brain. Neuroscience 180, 111-121. doi: 10.1016/j.neuroscience.2011. 02.023

McCullough, K. M., Daskalakis, N. P., and Ressler, K. J. (2018a). Cell-typespeci fi $\mathrm{c}$ interrogation of CeA Drd2 neurons to identify targets for pharmacological modulation of fear extinction. Transl. Psychiatry 8:164. doi: 10.1038/s41398-018-0190-y

McCullough, K. M., Morrison, F. G., Hartmann, J., Carlezon, W. A., and Ressler, K. J. (2018b). Quantified coexpression analysis of central amygdala subpopulations. eNeuro 5, 1-12. doi: 10.1523/ENEURO.0010-18.2018

Müller, T. D., Finan, B., Bloom, S. R., D’Alessio, D., Drucker, D. J., Flatt, P. R., et al. (2019). Glucagon-like peptide 1 (GLP-1). Mol. Metab. 30, 72-130. doi: 10.1016/j.molmet.2019.09.010

Qiu, L. R., Fernandes, D. J., Szulc-Lerch, K. U., Dazai, J., Nieman, B. J., Turnbull, D. H., et al. (2018). Mouse MRI shows brain areas relatively larger in males emerge before those larger in females. Nat. Commun. 9:2615. doi: 10.1038/s41467-018-04921-2

Secher, A., Vrang, N., Knudsen, L. B., Secher, A., Jelsing, J., Baquero, A. F., et al. (2014). The arcuate nucleus mediates GLP-1 receptor agonist liraglutidedependent weight loss. J. Clin. Invest. 124, 4473-4488. doi: 10.1172/JCI75276

Sisley, S., Gutierrez-Aguilar, R., Scott, M., D’Alessio, D. A., Sandoval, D. A., and Seeley, R. J. (2014). Neuronal GLP1R mediates liraglutide's anorectic but not glucose-lowering effect. J. Clin. Invest. 124, 2456-2463. doi: 10.1172/JCI 72434

Steinberg, E. E., Gore, F., Heifets, B. D., Taylor, M. D., Norville, Z. C., Beier, K. T., et al. (2020). Amygdala-midbrain connections modulate appetitive and aversive learning. Neuron 106, 1026-1043.e9. doi: 10.1016/j.neuron.2020.03.016

Terrill, S. J., Jackson, C. M., Greene, H. E., Lilly, N., Maske, C. B., Vallejo, S., et al. (2016). Role of lateral septum glucagon-like peptide 1 receptors in food intake. Am. J. Physiol. Regul. Integr. Comp. Physiol. 311, R124-R132. doi: 10.1152/ajpregu.00460.2015

Weera, M. M., Shackett, R. S., Kramer, H. M., Middleton, J. W., and Gilpin, N. W. (2021). Central amygdala projections to lateral hypothalamus mediate avoidance behavior in rats. J. Neurosci. 41, 61-72. doi: 10.1523/JNEUROSCI.0236-20.2020

Williams, D. L., Lilly, N. A., Edwards, I. J., Yao, P., Richards, J. E., and Trapp, S. (2018). GLP-1 action in the mouse bed nucleus of the stria 
terminalis. Neuropharmacology 131, 83-95. doi: 10.1016/j.neuropharm.2017. 12.007

Williams, E. K. K., Chang, R. B. B., Strochlic, D. E. E., Umans, B. D. D., Lowell, B. B. B., and Liberles, S. D. D. (2016). Sensory neurons that detect stretch and nutrients in the digestive system. Cell 166, 209-221. doi: 10.1016/j.cell.2016.05.011

Yu, K., da Silva, P. G., Albeanu, D. F., and Li, B. (2016). Central amygdala somatostatin neurons gate passive and active defensive behaviors. J. Neurosci. 36, 6488-6496. doi: 10.1523/JNEUROSCI.441915.2016

Conflict of Interest: The authors declare that the research was conducted in the absence of any commercial or financial relationships that could be construed as a potential conflict of interest.
Publisher's Note: All claims expressed in this article are solely those of the authors and do not necessarily represent those of their affiliated organizations, or those of the publisher, the editors and the reviewers. Any product that may be evaluated in this article, or claim that may be made by its manufacturer, is not guaranteed or endorsed by the publisher.

Copyright $\odot 2021$ Zeng, Cutts, Lopez, Kaur, Duran, Virkus and Hardaway. This is an open-access article distributed under the terms of the Creative Commons Attribution License (CC BY). The use, distribution or reproduction in other forums is permitted, provided the original author(s) and the copyright owner(s) are credited and that the original publication in this journal is cited, in accordance with accepted academic practice. No use, distribution or reproduction is permitted which does not comply with these terms. 